\title{
Релаксация энергии в квантовой точке на крае двумерного топологического изолятора
}

\author{
(C) Д.В. Хомицкий, Е.А. Лаврухина, А.А. Чубанов, Н. Нжийа \\ Национальный исследовательский Нижегородский государственный университет им. Н.И. Лобачевского, \\ 603950 Нижний Новгород, Россия \\ E-mail: khomitsky@phys.unn.ru
}

(Получена 27 апреля 2017 г. Принята к печати 12 мая 2017 г.)

Выполнен расчет скорости релаксации энергии для переходов с участием фононов в квантовых точках различного размера, созданных магнитными барьерами на крае двумерного топологического изолятора на базе квантовой ямы HgTe/CdTe. Рассматривается релаксация как в дискретный, так и в непрерывный спектр краевых состояний, а также в состояния непрерывного спектра объемного образца. Полученные результаты свидетельствуют о существовании области параметров структуры, обеспечивающей сравнительно медленную релаксацию энергии, что говорит о перспективности данных объектов, в том числе для создания новых типов твердотельных кубитов.

DOI: $10.21883 /$ FTP.2017.11.45111.25

\section{1. Введение}

В последнее десятилетие наблюдается значительный интерес к наноструктурам на основе топологических изоляторов (ТИ) [1-6]. В таких структурах, формируемых на базе как полупроводников, так и полуметаллов, уровень Ферми состояний объемного образца расположен в запрещенной зоне, что отвечает свойствам изолятора. При этом на крае ТИ, т.е. на двумерной поверхности в трехмерном ТИ или на одномерном крае для двумерного ТИ, могут существовать па́ры распространяющихся в противоположные стороны и эффективно переносящих ток состояний. Спин таких состояний жестко связан с направлением их распространения, поэтому они защищены от рассеяния на немагнитных примесях требованиями симметрии. Спектр их энергий пересекает запрещенную зону объемного материала и как функция квазиимпульса близок к линейной зависимости, поэтому такие краевые состояния называют безмассовыми фермионами, фермионами Дирака или фермионами Вейля, в зависимости от деталей строения спектра.

Для приложений ТИ в технологических задачах физики конденсированного состояния, в том числе задачах обработки информации и квантовых вычислений, необходимо разрабатывать компактные объекты на их базе, подобные квантовым точкам (КТ), которые могли бы выполнять роль элементарных ячеек для вычислений, т.е. кубитов. В последние годы было предложено несколько моделей таких КТ [7-10], сформированных на крае ТИ с помощью магнитных барьеров. Наличие магнитных барьеров в дополнение к электростатическим, создаваемым полем затвора, необходимо вследствие так называемого клейновского туннелирования, т. е. невозможности локализации состояний в ТИ без введения в гамильтониан массового члена, что эквивалентно наличию магнитного поля определенной ориентации [1-6]. В упомянутых работах [7-10] использовались модели с непроницаемыми магнитными барьерами, формирующими бесконечную последовательность уровней дискретно- го спектра. В нашей недавней работе [11] мы развили ранее созданные модели КТ для более реалистичных ситуаций проницаемых барьеров, формирующих мезоскопическую КТ с большим, но конечным числом уровней. Было показано, что при варьировании размеров КТ можно создавать структуру с почти произвольным количеством уровней дискретного спектра внутри нее, в том числе и с двумя уровнями, отстоящими друг от друга на значительном расстоянии. Это позволяет говорить о структуре как потенциально пригодной для создания кубита.

Для исследования вопроса о применимости такого объекта в том числе в качестве кубита, первостепенной является задача рассмотрения релаксационных свойств квантовых состояний. Необходимо рассматривать как релаксацию энергии, т.е. диагональных компонент матрицы плотности, так и релаксацию недиагональных компонент матрицы плотности. В настоящей работе мы делаем первый шаг к исследованию релаксационных свойств двухуровневой КТ на базе ТИ и рассчитываем скорость релаксации энергии при переходах с участием фононов. Такие переходы, как будет показано, приводят к достаточно высоким скоростям релаксации, что позволяет говорить об этом механизме релаксации как об одном из основных. Можно ожидать, что при высоких температурах его эффективность будет превосходить, например, вклад в рассеяние и релаксацию, обусловленный сверхтонким взаимодействием со спинами ядер решетки [12,13]. Расчет скорости релаксации при переходах между состояниями дискретного, а также дискретного и непрерывного спектров в КТ на крае ТИ требует знания фононного спектра в конкретной гетероструктуре. К настоящему времени последовательная и универсальная теория фононного спектра и электронфононного взаимодействия в гетероструктурах с краем, на котором существует фаза состояний ТИ, еще не создана. Для отдельных классов ТИ такие исследования выполнялись теоретически [14-20] и экспериментально [21-23]. Можно отметить результаты недавних экспе- 
риментов [24], в которых исследовался фононный спектр на поверхности объемного ТИ на базе $\mathrm{Bi}_{2} \mathrm{Se}_{3}$ методами рамановской спектроскопии. Было обнаружено, что переход от объемного образца к гетероструктуре с краем приводит к появлению новых ветвей в спектре фононов, связанных с поверхностными состояниями, однако их интенсивность в среднем на порядок уступает интенсивности фононных пиков от ветвей спектра в объеме образца. Это позволяет применить в качестве первого приближения для оценки скорости релаксации модель объемного спектра фононов в НgТе, параметры которого изучены с гораздо большей степенью надежности, в том числе экспериментально [25]. Кроме того, микроскопическая структура волновых функций краевых состояний отличается от структуры объемных прежде всего строением огибающих функций, в то время как блоховские функции, в основном определяющие значения параметров деформационного потенциала электрон-фононного взаимодействия, входят и в объемные, и в краевые состояния в схожих комбинациях, хотя и могут различаться набором коэффициентов [1-6]. Эти соображения позволяют в качестве первой оценки использовать надежно определенные параметры электрон-фононного взаимодействия для объемного материала $\mathrm{HgTe}$.

В данной работе представлены результаты расчета скорости релаксации энергии с участием фононов, полученные для одномерных квантовых точек различного продольного размера $L$, которые образованы магнитными барьерами на крае двумерного ТИ на базе квантовой ямы $\mathrm{HgTe} / \mathrm{CdTe}$. В разд. 2 описана модель квантовых состояний дискретного и непрерывного спектров, сформированных на крае ТИ в присутствии магнитных барьеров различной поляризации. В разд. 3 представлена модель для расчета скорости релаксации энергии с участием фононов, построенная в рамках модели деформационного потенциала с учетом оптических и акустических фононов, а также при учете взаимодействия с полярными оптическими фононами. В разд. 4 представлены полученные результаты расчета для скорости релаксации при низкой и при высокой температурах. Обсуждается относительный вклад различных каналов релаксации. В заключительном разд. 5 представлены выводы по работе и вопросы для будущих исследований.

\section{2. Модель квантовых состояний}

Для описания краевых состояний в присутствии магнитных барьеров, формирующих одномерную КТ на крае двумерного ТИ на базе КЯ $\mathrm{HgTe} / \mathrm{CdTe}$, нами был предложен гамильтониан, являющийся обобщением модели непроницаемых барьеров для случая барьеров конечной высоты и конечной прозрачности [11]:

$$
\begin{aligned}
H= & A k_{y} \sigma_{z}-M_{1} S(-y)\left(\sigma_{x} \cos \vartheta_{1}+\sigma_{y} \sin \vartheta_{1}\right) \\
& -M_{2} S(y-L)\left(\sigma_{x} \cos \vartheta_{2}+\sigma_{y} \sin \vartheta_{2}\right)
\end{aligned}
$$

Первое слагаемое в (1) отвечает гамильтониану Дирака-Вейля для одномерных состояний, распространяющихся вдоль края ТИ, с параметром

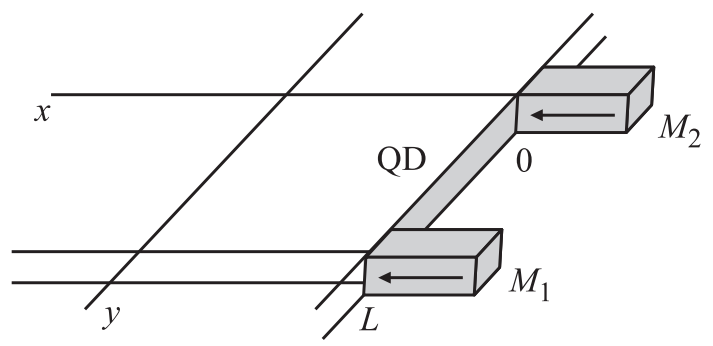

Рис. 1. Схема квантовой точки (область QD), сформированной между магнитными барьерами (параллелепипеды со стрелками) с варьируемыми амплитудами $\left(M_{1,2}\right)$ и углами ориентации $\left(\vartheta_{1,2}\right)$, образованной на крае топологического изолятора на основе квантовой ямы $\mathrm{HgTe} / \mathrm{CdTe}$.

$A=0.36$ эВ $\cdot$ нм [1-6]. Второе и третье слагаемые с матрицами Паули $\sigma_{x}, \sigma_{y}$, отвечают вкладам от магнитных барьеров с амплитудами $M_{1,2}$, намагниченности которых ориентированы в плоскости $x y$ под углами $\vartheta_{1,2}$ к оси $x$, а сами барьеры располагаются вдоль направления $y$ на расстоянии $L$ друг от друга и аппроксимируются ступенчатой функцией $S(x)=1$ при $x>0, S(x)=0$ при $x<0$. Значения высот барьеров $M_{1,2}$ выбирались равными или несколько меньшими половины ширины запрещенной зоны в спектре объемных состояний двумерного электронного газа для КЯ шириной 8-9 нм, составляющей $\sim 40$ мэВ [1-6]. Предложенная схема КТ показана на рис. 1.

Собственные функции для гамильтониана (1) представляют собой двухкомпонентные спиноры, координатная часть которых имеет вид стоячих волн между барьерами и экспоненциально затухает вглубь них. Волновая функция обозначена нами как $\psi_{\mathrm{QD}}$ в области КТ и $\psi_{y<0}, \psi_{y>0}$ в области левого и правого барьеров соответственно:

$$
\begin{gathered}
\psi_{\mathrm{QD}}=\left(\begin{array}{c}
C_{1} e^{i E y / A} \\
C_{2} e^{i E y / A}
\end{array}\right), \\
\psi_{y<0}=B\left(\begin{array}{c}
1 \\
-\frac{i \sqrt{M_{1}^{2}-E^{2}}+E}{M_{1}} e^{i \vartheta_{1}}
\end{array}\right) \exp \left(-\frac{\sqrt{M_{1}^{2}-E^{2}}}{A} y\right), \\
\psi_{y>L}=D\left(\begin{array}{c}
1 \\
-\frac{i \sqrt{M_{2}^{2}-E^{2}}+E}{M_{2}} e^{i \vartheta_{2}}
\end{array}\right) \exp \left(-\frac{\sqrt{M_{2}^{2}-E^{2}}}{A} y\right) .
\end{gathered}
$$

Коэффициенты $C_{1}, C_{2}, B, D$ определяются из граничных условий, описывающих непрерывность волновой функции на границах КТ и барьеров:

$$
\left\{\begin{array}{l}
\psi(y=0-)=\psi(y=0+) \\
\psi(y=L-)=\psi(y=L+)
\end{array} .\right.
$$

После подстановки спинорных волновых функций (2)-(4) в систему (5) мы приходим к системе 
линейных однородных уравнений для неизвестных коэффициентов $C_{1}, C_{2}, B, D$. Условие существования нетривиального решения этой системы определяет секулярное уравнение для энергии $E$ дискретных уровней в КЯ. После его численного решения для каждого уровня $E_{n}$ определяются волновые функции (2)-(4). Зависимость положения энергетических уровней в КТ от ее ширины $L$ для случая параллельной ориентации магнитных барьеров $\vartheta_{1}=\vartheta_{2}=0$ с амплитудой $M_{1}=M, M_{2}=M$, где $M=20$ мэВ, показана на рис. 2 .

Видно, что при $L<70$ нм в КТ находятся лишь два дискретных уровня $|1\rangle$ и $|2\rangle$ по обе стороны от точки Дирака $E=0$, т. е. имеется двухуровневая система. Если уровень Ферми находится вблизи точки Дирака $E=0$, как это имеет место во многих ТИ [1-6], то уровень $|1\rangle$ на рис. 2 в равновесном состоянии при низких температурах будет занят, а уровень $|2\rangle$ будет свободен. При возбуждении внешним источником, например, импульсом электрического поля с несущей частотой, равной межуровневому расстоянию, возможны эффективные переходы с уровня $|1\rangle$ на уровень $|2\rangle$. После снятия возмущения с учетом взаимодействия с фононами следует ожидать переходов с дискретного уровня $|2\rangle$ на дискретный уровень $|1\rangle$, что отвечает релаксации энергии.

Следует отметить, что, помимо уровней дискретного спектра в системе с гамильтонианом (1) существуют и краевые состояния непрерывного спектра, в которые также могут происходить переходы с каждого из уровней дискретного спектра при участии фононов. Расчет таких состояний аналогичен расчету состояний дискретного спектра. Будем обозначать волновую функцию непрерывного спектра как $\tilde{\psi}$. По аналогии с (2)-(4), двухкомпонентные спиноры в этом случае записываются следующим образом:

$$
\tilde{\psi}_{\mathrm{QD}}=\left(\begin{array}{c}
\tilde{C}_{1} e^{i E_{y} / A} \\
\tilde{C}_{2} e^{-i E_{y} / A}
\end{array}\right),
$$

$$
\begin{aligned}
\tilde{\psi}_{y<0} & =\tilde{B}_{1}\left(\begin{array}{c}
1 \\
\frac{-E+\sqrt{E^{2}-M_{1}^{2}}}{M_{1}} e^{i \vartheta_{1}}
\end{array}\right) \exp \left(i \frac{\sqrt{E^{2}-M_{1}^{2}}}{A} y\right) \\
& +\tilde{B}_{2}\left(\begin{array}{c}
1 \\
\frac{-E-\sqrt{E^{2}-M_{1}^{2}}}{M_{1}} e^{i \vartheta_{1}}
\end{array}\right) \exp \left(-i \frac{\sqrt{E^{2}-M_{1}^{2}}}{A} y\right),
\end{aligned}
$$

$$
\begin{aligned}
\tilde{\psi}_{y>L} & =\tilde{D}_{1}\left(\begin{array}{c}
1 \\
\frac{-E+\sqrt{E^{2}-M_{2}^{2}}}{M_{2}} e^{i \vartheta_{2}}
\end{array}\right) \exp \left(i \frac{\sqrt{E^{2}-M_{2}^{2}}}{A} y\right) \\
& +\tilde{D}_{2}\left(\begin{array}{c}
1 \\
\frac{-E-\sqrt{E^{2}-M_{2}^{2}}}{M_{2}} e^{i \vartheta_{2}}
\end{array}\right) \exp \left(-i \frac{\sqrt{E^{2}-M_{2}^{2}}}{A} y\right) .
\end{aligned}
$$

Коэффициенты $\tilde{C}_{1}, \tilde{C}_{2}, \tilde{B}_{1}, \tilde{B}_{2}, \tilde{D}_{1}, \tilde{D}_{2}$, вновь определяются из граничных условий (5). Однако в случае

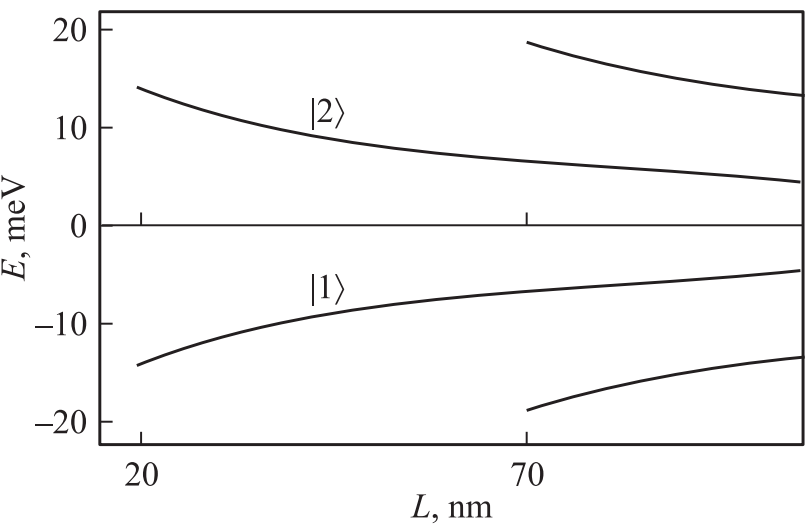

Рис. 2. Зависимость уровней энергии в квантовой точке от ее ширины $L$ при параллельно ориентации магнитных барьеров. При $L<70$ нм образуется одна пара дискретных уровней $|1\rangle$ и $|2\rangle$ по обе стороны от точки Дирака $E=0$.

состояний непрерывного спектра у нас имеется шесть, а не четыре неизвестных коэффициента. Два из них могут быть заданы произвольно, что отвечает волне, выходящей из области слева от КТ или из области справа от КТ. Остальные четыре коэффициента будут найдены из системы (5). Секулярное уравнение для энергии $E$ в этом случае отсутствует, и единственным условием существования таких состояний, как это видно из структуры спиноров (6)-(8), является превышение энергией $E$ амплитуды магнитных барьеров $M_{1}$ и $M_{2}$, т. е. выполнение условия

$$
|E|>M_{1,2} \text {. }
$$

Далее в работе нами рассчитана скорость переходов при релаксации с участием фононов как между состояниями (2)-(4) дискретного спектра, так и с уровней дискретного спектра в краевые состояния непрерывного спектра (6)-(8), энергия которых удовлетворяет условию (9).

\section{3. Схема расчета скорости релаксации}

Для получения оценки скорости релаксации энергии, как это обсуждалось во Введении, нами будет использована простейшая модель электрон-фононного взаимодействия в приближении деформационного потенциала, т.е. микрополя [26]. Кроме того, дополнительно будет вычислена скорость релаксации и при учете макрополя, т.е. полярных оптических фононов [26], но лишь для расчета переходов между состояниями дискретного спектра $|1\rangle$ и $|2\rangle$, упомянутыми в предыдущем разделе. Далее будет показано, что при низких температурах такие переходы являются доминирующими. Кроме того, учет полярных фононов для переходов в непрерывный спектр требует более точных сведений о параметрах фононов во всем объеме гетероструктуры $\mathrm{HgTe} / \mathrm{CdTe}$ с фазой ТИ, которые к настоящему времени еще не определены. 


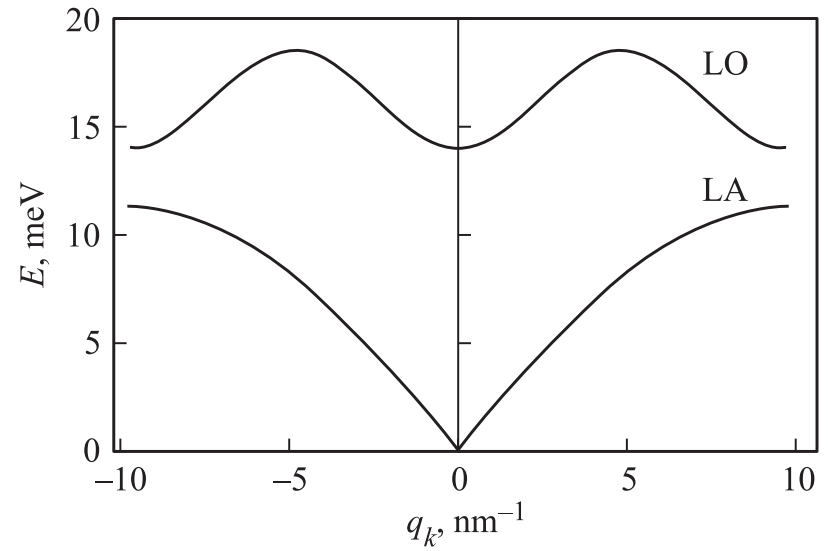

Рис. 3. Аналитическое изотропное приближение для спектра фононов в НgТе. Показана ветвь продольных оптических фононов LO и ветвь продольных акустических фононов LA.

Расстояния между дискретными уровнями $|1\rangle$ и $|2\rangle$, показанные на рис. 2 при типичных значениях ширины КТ $L=40-60 \mathrm{Hм,} \mathrm{составляют} \mathrm{15-20мэВ.} \mathrm{Такой} \mathrm{же}$ порядок имеют и расстояния от любого уровня дискретного спектра до состояний непрерывного спектра, начинающихся при энергиях $|E|>M$, где $M=20$ мэВ. Это значит, что доминирующая роль в переходах принадлежит продольным оптическим фононам, энергия которых в $\mathrm{HgTe}$, в пространственной области которого расположены краевые состояния, попадает в указанный интервал. Модель спектра продольных фононов в объемном HgТе построена нами как максимально близкая к экспериментальным данным из работы [25]. В упрощенной форме, допускающей аналитическое описание в пределе изотропной зависимости частоты от компонент волнового вектора $\omega\left(q_{1}, q_{2}, q_{3}\right)$, она показана на рис. 3 для какого-либо из направлений $q_{k}$. Приближение изотропного спектра для продольных фононов находится в хорошем согласии с экспериментальными данными [25]. Нами рассматривается взаимодействие с продольными оптическими фононами (LO) и с продольными акустическими фононами (LA), которые вносят основной вклад в релаксацию, поскольку энергии поперечных фононов уступают энергии продольных. Учет акустических фононов необходим при высоких температурах, когда становятся эффективными переходы с поглощением фононов с уровня $|2\rangle$ дискретного спектра в КТ в краевые состояния непрерывного спектра с энергией $E>M$.

Наличие точек с равной нулю групповой скоростью на ветви LO в точках максимума частоты фонона (см. рис. 3) приводит к особенностям плотности состояний в фононном спектре. Это, как показывают наши расчеты, дает увеличение скорости релаксации, если параметры КТ таковы, что расстояние между уровнями $|1\rangle$ и $|2\rangle$ дискретного спектра в точности соответствует максимуму частоты LO фонона $\omega_{\mathrm{LO}}=2.82 \cdot 10^{13} \mathrm{c}^{-1}$. На практике, однако, точное выполнение этого условия технологически мало вероятно и практически нецеле- сообразно, поэтому данная особенность в дальнейшем нами рассматриваться не будет.

Скорость релаксации энергии $\Gamma_{i j}$ между состояниями $|i\rangle$ и $|j\rangle$ с энергиями $E_{i}, E_{j}$ при переходах с участием фонона с волновым вектором $\left(q_{1}, q_{2}, q_{3}\right)$ и энергией $\hbar \omega_{q}$ в кристалле объемом $V$ определяется как [26]

$\Gamma_{i j}=\frac{2 \pi}{\hbar} \int \frac{V d^{3} q}{(2 \pi)^{3}}\left|M_{i j}^{q}\right|^{2}\left(N_{q}+\frac{1}{2} \pm \frac{1}{2}\right) \delta\left(E_{i}-E_{j} \mp \hbar \omega_{q}\right)$,

где верхний знак отвечает испусканию, а нижний поглощению фононов, описываемых равновесным распределением Планка $N_{q}$. Из (10) следует, что для нашей структуры при низких температурах, $1-5 \mathrm{~K}$, когда для типичных энергий переходов 10-20 мэВ среднее число фононов $N_{q} \ll 1$, будут доминировать процессы испускания фононов, т.е. переходы „сверху вниз“. Лишь для более высоких температур, $50-100 \mathrm{~K}$, могут быть существенны переходы вида „снизу вверх“ с поглощением фононов. Матричный элемент деформационного потенциала для оптических (LO) и акустических (LA) фононов вычисляется соответственно как [26]

$$
\begin{aligned}
\left|M_{i j}^{q}\right|^{2} & =\frac{\hbar a C_{\mathrm{op}}^{2}}{V 2 M \omega_{s}}\left|\left\langle i\left|e^{i \mathbf{q r}}\right| j\right\rangle\right|^{2}, \\
\left|M_{i j}^{q}\right|^{2} & =\frac{\hbar a^{3} C_{\mathrm{ac}}^{2}}{V 2 M \omega_{q}}\left|\left\langle i\left|e^{i \mathbf{q r}}\right| j\right\rangle\right|^{2} .
\end{aligned}
$$

Здесь $a=0.646$ нм есть постоянная решетки в $\mathrm{HgTe}$, $M=1.29 \cdot 10^{-22} \Gamma$ есть приведенная масса атомов в элементарной ячейке, $\omega_{s}$ или $\omega_{q}$ обозначают частоту оптического фонона при $q=0$ или частоту акустических фононов соответственно. Эти параметры, а также константы деформационного потенциала для ТИ на базе КЯ $\mathrm{HgTe} / \mathrm{CdTe} C_{\text {op }}=20{ } \mathrm{~B}$ и $C_{\mathrm{ac}}=5$ эВ были взяты нами из [20]. Кроме того, при переходах между дискретными уровнями в КТ нами рассчитывалась скорость релаксации при учете макрополя от взаимодействия с полярными оптическими фононами (РО), взаимодействие с которыми разрешено симметрией решетки $\mathrm{HgTe}$ со структурой цинковой обманки. В этом случае матричный элемент электрон-фононного взаимодействия равен [26]

$$
\begin{aligned}
& \left|M_{i j}^{q}\right|^{2}=\frac{e^{2} \hbar a^{3} C_{P}^{2}}{V 2 M \omega_{s}}\left|\left\langle i\left|\frac{e^{i \mathbf{q r}}}{i q}\right| j\right\rangle\right|^{2}, \\
& C_{P}=\left[\frac{4 \pi M}{a^{3}}\left(\frac{1}{\varepsilon_{\infty}}-\frac{1}{\varepsilon_{0}}\right)\right]^{1 / 2} \omega_{\mathrm{LO}},
\end{aligned}
$$

где $\omega_{\mathrm{LO}}=2.82 \cdot 10^{13} \mathrm{c}^{-1}$ есть предельная частота продольного оптического фонона, $\varepsilon_{0}=20$ и $\varepsilon_{\infty}=14$ есть статическая диэлектрическая проницаемость и диэлектрическая проницаемость на больших частотах соответственно [20]. Для переходов из дискретного в непрерывный спектр канал с взаимодействием (13) нами не учитывался, так как пока не известны точные характеристики макроскопического поля фононов в ТИ на 
базе КЯ $\mathrm{HgTe} / \mathrm{CdTe}$ во всем объеме гетероструктуры, в то время как переходы между дискретными уровнями отвечают локальному взаимодействию с фононами в узкой краевой области образца.

\section{4. Результаты расчета}

\section{1. Скорость релаксации при низкой температуре}

Результаты расчета скорости релаксации энергии (10) показаны на рис. 4 для низкой температуры, $T=4 \mathrm{~K}$, в полулогарифмическом масштабе как функция ширины КТ $L$.

При положении уровня Ферми вблизи точки Дирака $E=0$, как это видно из рис. 2, при возбуждении внешним полем носителей на уровень $|2\rangle$ после прекращения действия возмущения будет доминировать переход на нижний уровень $|1\rangle$ дискретного спектра с испусканием оптического фонона. Дискретные уровни на протяжении процесса перехода предполагаются выведенными из термодинамического равновесия. Переходы с поглощением фонона в вышележащие состояния непрерывного спектра при низкой температуре подавлены, поскольку $N_{q} \ll 1$. Также в силу принципа Паули сильно подавлены переходы с нижнего уровня $|1\rangle$ в состояния непрерывного спектра с отрицательными энергиями относительно точки Дирака $E=0$, поскольку при $T=4 \mathrm{~K}$ эти состояния почти полностью заполнены. При этом $\left(1-f_{j}\right) \approx 0$, где $f_{j}$ есть функция Ферми для конечных состояний в непрерывном спектре, которые предполагаются находящимися в состоянии термодинамического равновесия. Мы отдельно рассчитываем скорость релаксации (10) с матричными элементами (11) для деформационного потенциала оптических фононов и с матричными элементами (13) для полярных оптических фононов.

Из рис. 4 можно сделать вывод, что скорость релаксации почти монотонно растет с шириной КТ $L$, и для $L=60$ нм она на 6 порядков превосходит значение для $L=42$ нм. Объяснение этому эффекту заключается в расположении дискретных уровней в КТ на рис. 2, а также в зависимости матричного элемента (11) от волнового вектора фонона $\left(q_{1}, q_{2}, q_{3}\right)$. С ростом $L$ уровни сближаются, и переходы происходят с меньшими значениями энергии LO фонона $\hbar \omega_{q}$. Следуя ходу дисперсионной кривой для LO фононов на рис. 3 в области $q_{k}<5$ нм$^{-1}$, при уменьшении энергии фонона в переходах участвуют фононы со все меньшими значениями модуля волнового вектора $q_{k}$. Здесь $k=1,2,3$, так как мы приняли для оценки изотропную модель спектра фононов. Матричный же элемент (11), как это следует из аналитического и численного расчетов, является быстро спадающей функцией волнового вектора фононной экспоненты. В результате скорость релаксации тем выше, чем более длинноволновые фононы участвуют в переходах, т.е. чем более широкая КТ рассматривается. Второй вывод, следующий из рис. 4 , заключается в

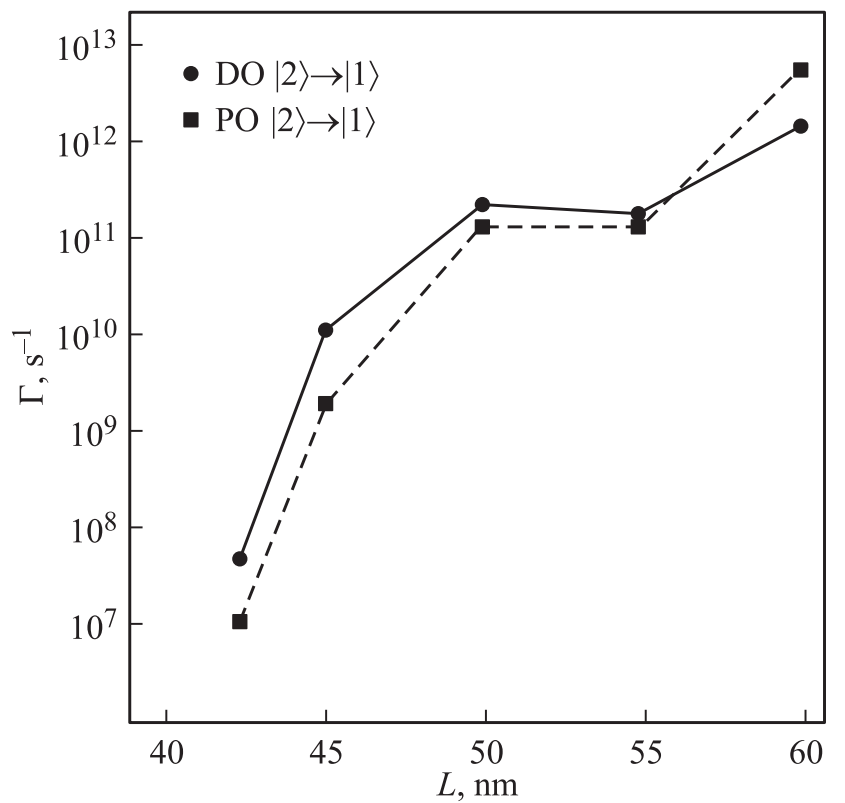

Рис. 4. Скорость релаксации между уровнями дискретного спектра $|2\rangle \rightarrow|1\rangle$ для температуры $T=4 \mathrm{~K}$. Показаны вклады оптических фононов в приближении деформационного потенциала (DO) и от взаимодействия с полярными оптическими фононами (PO).

качественно одинаковом поведении и величине скорости релаксации в канале взаимодействия с оптическими фононами от микрополя, т. е. деформационного потенциала (DO), и от макрополя, т. е. полярных фононов (PO). Такой результат позволяет говорить о качественно схожей оценке скорости переходов в целом, в независимости от характера участвующих в нем оптических фононов. Еще одним вопросом, возникающим при анализе рис. 4, является вопрос об экстраполяции результатов на КТ других размеров, в особенности на более узкие КТ, с $L<42$ нм. Такое граничное значение ширины КТ в модели спектра уровней на рис. 2 и модели фононного спектра на рис. 3 обусловлено максимальной энергией оптических фононов в $\mathrm{HgTe}$, которая в нормальных условиях составляет $\sim 18.5$ мэВ [25]. Если КТ имеет ширину $<42$ нм, то уровни $|1\rangle$ и $|2\rangle$ отстоят друг от друга на величину энергии, превосходящую максимальную энергию фононов в $\mathrm{HgTe}$. Это означает, что в более узких КТ канал релаксации через оптические фононы будет малоэффективен, особенно при низких температурах, т.е. время жизни в возбужденном состоянии $|2\rangle$ сильно возрастет. Однако технологические сложности процессов формирования наноразмерных объектов с включением в них магнитов так же микро- и нанометровых размеров делают изготовление таких КТ все более затруднительными при уменьшении размера КТ. Таким образом, для приложений предложенной в нашей предыдущей работе [11] и в данной работе модели КТ в качестве двухуровневой системы с медленной релаксацией энергии необходим технологический ком- 
промисс, сочетающий малые размеры КТ, в которой релаксация уменьшена или подавлена, и существующие технологические возможности.

\section{2. Релаксация в непрерывный спектр при высокой температуре}

При повышении температуры от $T=4$ до $77 \mathrm{~K}$ канал релаксации между дискретными уровнями с испусканием фонона, описанный в предыдущем разд. 4.1, не претерпевает существенных изменений, поскольку значение фактора $\left(N_{q}+1\right)$ в (10) для оптических фононов с энергией 14-18 мэВ остается близким к единице. Поэтому мы обратим свое внимание на другие каналы релаксации, открывающиеся при повышении температуры, а именно на релаксацию при переходах с уровней $|1\rangle$ и $|2\rangle$ дискретного спектра КТ в состояния непрерывного спектра. Нами рассмотрено пять таких каналов, и далее они будут кратко описаны. Результаты для скорости релаксации в этих каналах сведены воедино на рис. 5 как функция ширины КТ $L$.

4.2.1. Скорость переходов с верхнего уровня $|2\rangle$ В краевые состояния непрерывного спектра с поглощением оптических фононов (6)-(8) обозначена на рис. 5 как LO $|2\rangle \rightarrow \mathrm{Up}($ Edge $)$. Такие процессы с поглощением оптического фонона эффективны лишь при высокой температуре, составляющей в нашем примере $77 \mathrm{~K}$, поскольку лишь в этом случае имеется существенное среднее число оптических фононов. Поскольку краевые состояния непрерывного спектра существуют с любыми энергиями $E>M$, где $M=20$ мэВ есть высота магнитного барьера, и начинаются сразу же над краем барьера, то для всех рассмотренных значений ширины КТ $L$ эти переходы будут вносить существенный вклад в скорость релаксации при высокой температуре, составляющий в среднем $10^{12} \mathrm{c}^{-1}$.

4.2.2. Скорость переходов с нижнего уровня $|\mathbf{1}\rangle$ в краевые состояния непрерывного спектра с испусканием оптических фононов показана на рис. 5 как LO $|1\rangle \rightarrow$ Down(Edge). Такие переходы с испусканием оптического фонона становятся эффективными при $T=77 \mathrm{~K}$, поскольку существенная часть краевых состояний под уровнем Ферми с энергией $E<-M$ при высокой температуре является свободной, и множитель $\left(1-f_{j}\right)$, определяющий вероятность этого, уже существенно отличается от нуля.

Мы видим, что, как и в предыдущем разделе, в этом случае скорость релаксации с нижнего уровня слабо зависит от размера КТ и достигает существенных значений $10^{11}-10^{12} \mathrm{c}^{-1}$. Это означает, что при высоких температурах становится эффективной релаксация не только с „возбужденного“ уровня дискретного спектра $|2\rangle$, но и с „основного“ уровня $|1\rangle$, если принимать во внимание краевые состояния непрерывного спектра. Такой канал релаксации также необходимо учитывать при создании схем кубитов на данной КТ, работающих при высоких температурах.

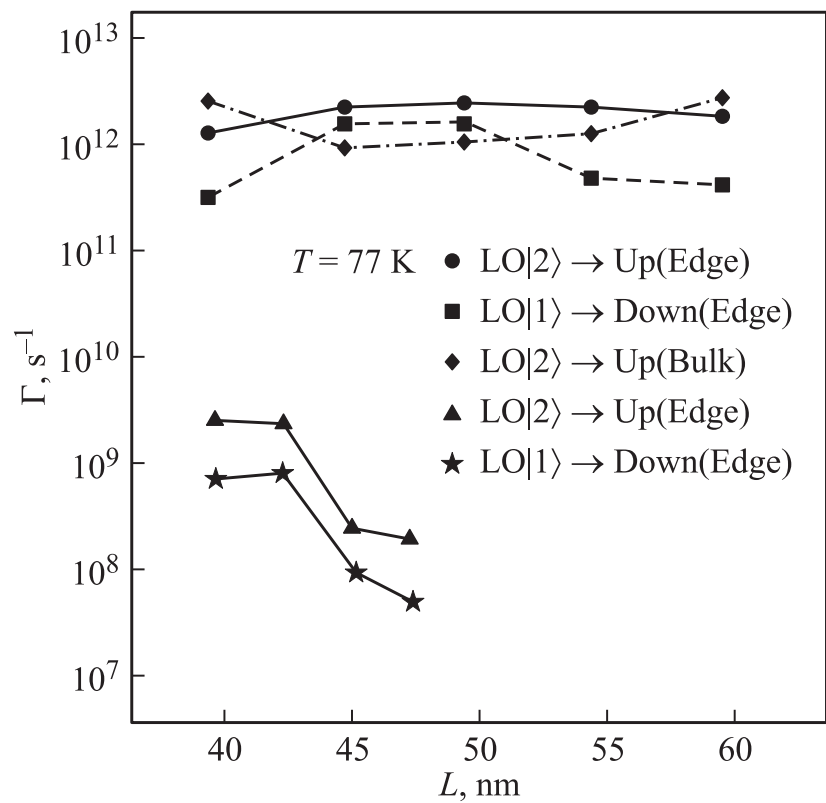

Рис. 5. Скорость релаксации между состояниями дискретного и непрерывного спектров для $T=77 \mathrm{~K}$.

4.2.3. Скорость переходов верхнего уровня $|2\rangle$ В состояния непрерывного спектра объемного материала с поглощением оптических фононов показана на рис. 5 и обозначена как LO $|2\rangle \rightarrow \mathrm{Up}(\mathrm{Bulk})$. Состояния объемного материала представляют собой двумерный электронный газ в КЯ $\mathrm{HgTe} / \mathrm{CdTe}$, на крае которого образуется спектр краевых состояний. Энергетический спектр и вид волновых функций двумерного электронного газа в такой структуре хорошо известен и взят нами из работ [1-6] с целью оценки скорости релаксации в такие состояния с верхнего дискретного уровня в КТ. Упрощенно, состояния объемного образца могут быть описаны как плоские волны в плоскости двумерного электронного газа $(x y)$, локализованные в поперечном направлении $z$ роста КЯ. Спинорный характер этих состояний отражается преимущественно в многокомпонентной форме вектора-столбца в базисе блоховских функций, содержащего функции $\left\{f_{i}(z)\right\}$, описывающие их локализацию в КЯ. Для нашей задачи детальная структура этого спинора не является существенной, так как все его компоненты локализованы в области КЯ и этого оказывается достаточно для оценки матричных элементов (11). Поскольку процесс перехода с уровня $|2\rangle$ в состояния непрерывного спектра идет с поглощением фонона, он становится существенным лишь при высоких температурах. Из рис. 5 видно, что его скорость слабо зависит от размера КТ и достигает существенной величины $10^{12} \mathrm{c}^{-1}$. Что касается „симметричного“ процесса ухода с нижнего уровня $|1\rangle$ в состояния объемного образца с отрицательными энергиями (ниже точки Дирака), то такие процессы не разрешены, поскольку нижняя половина спектра объемных состояний в КЯ $\mathrm{HgTe} / \mathrm{CdTe}$ отстоит от точки $E=0$ дальше, 
чем верхняя половина [1-6], и энергии даже оптических фононов для реализации такого перехода оказывается недостаточно.

4.2.4. Скорость переходов с поглощением акустических фононов с уровня $|2\rangle$ в краевые состояния непрерывного спектра показана на рис. 5 и обозначена как $\mathrm{LA}|2\rangle \rightarrow \mathrm{Up}($ Edge $)$. При $T=77 \mathrm{~K}$ значение $N_{q}$ в (10) для акустических фононов становится существенным, и переходы с поглощением акустического фонона в краевые состояния непрерывного спектра также приобретают заметную эффективность. Поскольку энергия продольных акустических фононов в $\mathrm{HgTe}$ ограничена величиной $\sim 11.5$ мэВ [25] (см. также рис. 3), указанные переходы с их участием эффективны лишь для КТ с $L=40-47 \mathrm{HM}$, поскольку для более широких КТ дискретные уровни располагаются ближе к точке Дирака $E=0$ и энергии акустических фононов оказывается недостаточно, чтобы осуществить переход в непрерывный спектр с энергией $E>M$. По этой причине неэффективны и переходы в состояния объемного материала. Кроме того, максимальная энергия акустических фононов достигается при больших волновых числах, что приводит к сильному уменьшению амплитуды матричного элемента (12). Все это приводит к тому, что скорость релаксации на акустических фононах существенно, на 3-4 порядка, ниже скорости релаксации на оптических фононах, как это видно из рис. 5 , и составляет $\sim\left(10^{8}-10^{9}\right) \mathrm{c}^{-1}$. Существенным является возрастание этой скорости с уменьшением размера КТ, что может быть дополнительным ограничивающим фактором при использовании очень малых значений $L$ для приложений таких КТ.

4.2.5. Скорость переходов с испусканием акустических фононов с уровня $|1\rangle$ в краевые состояния непрерывного спектра показана на рис. 5 и обозначена как LA $|1\rangle \rightarrow$ Down(Edge). При $T=77 \mathrm{~K}$ значение множителя $\left(1-f_{j}\right)$, как это обсуждалось в разд. 4.2.2, достаточно велико, чтобы учитывать переходы с нижнего уровня $|1\rangle$ в краевые состояния непрерывного спектра с испусканием акустического фонона. К расчету их величины применимы все соображения разд. 4.2.4. Скорость релаксации в этом канале, как видно из рис. 5 , составляет $10^{7}-10^{9} \mathrm{c}^{-1}$ и вновь возрастает с уменьшением размера КТ.

\section{5. Заключение}

В работе выполнен расчет скорости релаксации энергии при переходах с участием фононов для одномерных квантовых точек различного продольного размера $L$, образованных магнитными барьерами на крае двумерного топологического изолятора на базе квантовой ямы $\mathrm{HgTe} / \mathrm{CdTe}$. Полученные результаты свидетельствуют о существовании области параметров структур, отвечающих небольшим квантовым точкам с продольным размером $L<42 \mathrm{Hм}$, где может быть обеспечена более медленная релаксация энергии при низкой температуре,
$T=4 \mathrm{~K}$, по сравнению с квантовыми точками с размером $L>42$ нм. Наличие такой области говорит о принципиальной перспективности данных объектов, в том числе для создания новых типов кубитов. Дальнейшие исследования требуют в первую очередь расчетов времени релаксации для недиагональных компонент матрицы плотности, а также уточнения параметров деформационного потенциала и потенциала взаимодействия с полярными фононами для конкретных типов гетероструктур с фазой топологического изолятора. Кроме того, необходимо прояснить роль поверхностных фононных мод в их возможном вкладе в релаксацию, а также более детально учесть взаимодействие с состояниями в объеме гетероструктуры. Указанные вопросы никоим образом не ограничивают круг тем для возможных будущих исследований в этой перспективной области.

Авторы выражают признательность А.М. Сатанину, В.А. Бурдову и А.А. Конакову за полезные обсуждения.

Разд. 2 работы выполнен при поддержке гранта РФФИ № 16-57-51045-НИФ_а. Разд. 3, 4, 5 работы выполнены при поддержке гранта в рамках проектной части государственного задания Министерства образования и науки РФ 3.3026.2017/ПЧ.

\section{Список литературы}

[1] M.Z. Hasan, C.L. Kane. Rev. Mod. Phys., 82, 3045 (2010).

[2] X.-L. Qi, S.-C. Zhang. Rev. Mod. Phys., 83, 1057 (2011).

[3] B.A. Bernevig, Topological Insulators and Topological Superconductors (Princeton University Press, Princeton, 2013).

[4] Topological Insulators. Fundamentals and Perspectives, ed. by F. Ortmann, S. Roche, and S.O. Valenzuela (Wiley-VCH Verlag, Weinheim, 2015).

[5] B.A. Bernevig, T.L. Hughes, S.-C. Zhang. Science, 314, 1757 (2006).

[6] M. König, H. Buhmann, L.W. Molenkamp, T.L. Hughes, C.-X. Liu, X.-L. Qi, S.-C. Zhang. J. Phys. Soc. Jpn., 77, 031007 (2008).

[7] A. Kundu, A. Zazunov, A.L. Yeyati, T. Martin, R. Egger. Phys. Rev. B, 83, 125429 (2011).

[8] C. Timm. Phys. Rev. B, 86, 155456 (2012).

[9] G. Dolcetto, N. Traverso Ziani, M. Biggio, F. Cavaliere, M. Sassetti. Phys. Rev. B, 87, 235423 (2013).

[10] G.J. Ferreira, D. Loss. Phys. Rev. Lett., 111, 106802 (2013).

[11] D.V. Khomitsky, A.A. Chubanov, A.A. Konakov. ЖЭТФ, 150 (6), 1200 (2016).

[12] A.M. Lunde, G. Platero. Phys. Rev. B, 88, 115411 (2013).

[13] S.A. Tarasenko, G. Burkard. Phys. Rev. B, 94, 045309 (2016).

[14] E.M. Sheregii, J. Cebulski, A. Marcelli, M. Piccinini. Phys. Rev. Lett., 102, 045504 (2009).

[15] P. Thalmeier. Phys Rev. B, 83, 125314 (2011).

[16] S. Giraud, R. Egger. Phys. Rev. B, 83, 245322 (2011).

[17] B.-T. Wang, P. Zhang. Appl. Phys. Lett., 100, 082109 (2012).

[18] S. Giraud, A. Kundu, R. Egger. Phys. Rev. B, 85, 035441 (2012).

[19] V. Parente, A. Tagliacozzo, F. von Oppen, F. Guinea. Phys. Rev. B, 88, 075432 (2013). 
[20] K. Saha, I. Garate. Phys. Rev. B, 89, 205103 (2014).

[21] X. Zhu, L. Santos, C. Howard, R. Sankar, F.C. Chou, C. Chamon, M. El-Batanouny. Phys. Rev. Lett., 108, 185501 (2012).

[22] Z.-H. Pan, A.V. Fedorov, D. Gardner, Y.S. Lee, S. Chu, T. Valla. Phys. Rev. Lett., 108, 187001 (2012).

[23] E. Golias, J. Sánchez-Barria. Phys. Rev. B, 94, 161113(R) (2016).

[24] H.-H. Kung, M. Salehi, I. Boulares, A.F. Kemper, N. Koirala, M. Brahlek, P. Lošták, C. Uher. R. Merlin, X. Wang, S.-W. Cheong, S. Oh, G. Blumberg. arXiv:1611.05926 (2016).

[25] S. Radescu, A. Mujica, R.J. Needs. Phys. Rev. B, 80, 144110 (2009).

[26] В.Ф. Гантмахер, И.Б. Левинсон. Рассеяние носителей тока в металлах и полупроводниках (М., Наука, 1984) гл. 3.

Редактор Л.В. Шаронова

\section{Energy relaxation in a quantum dot at the edge of two-dimensional topological insulator}

D.V. Khomitsky, E.A. Lavrukhina,

A.A. Chubanov, N. Njiya

National Research Lobachevsky State University

of Nizhny Novgorod,

603950 Nizhny Novgorod, Russia

Abstract The calculation of energy relaxation rates due to transitions involving phonons is performed for quantum dots of variable size which are formed by magnetic barriers at the edge of two-dimensional topological insulator based on a $\mathrm{HgTe} / \mathrm{CdTe}$ quantum well. The relaxation both into the discrete and continuum spectrum of edge states is considered, as well as into the continuum spectrum of the bulk material. The obtained results demonstrate the existence of the area of system parameters which provide relatively slow energy relaxation, indicating that the considered objects are promising for design, among other things, of novel types of solid state qubits. 